\title{
Development of a Remote Controlled Alert System for Automobile Access into Premises
}

\author{
Daniyan Ilesanmi, Adeodu Adefemi, Kayode Joseph, Asibe Justice, Aderoba Adeyemi \\ Department of Mechanical and Mechatronics Engineering, Afe Babalola University, Ado Ekiti, Nigeria

\section{Email address:} \\ afolabiilesanmi@yahoo.com (Daniyan I.), adeoduac@abuad.edu.ng (Adeodu A.), jkayode2011@yahoo.com (Kayode J.), \\ babalotujama@yahoo.com (Aderoba A.)
}

\section{To cite this article:}

Daniyan Ilesanmi, Adeodu Adefemi, Kayode Joseph, Asibe Justice, Aderoba Adeyemi. Development of a Remote Controlled Alert System for Automobile Access into Premises. Automation, Control and Intelligent Systems. Vol. 5, No. 5, 2017, pp. 61-66.

doi: 10.11648/j.acis.20170505.11

Received: April 22, 2017; Accepted: August 23, 2017; Published: September 22, 2017

\begin{abstract}
There have been various developments of security system such as computer security systems which prevent unauthorized access to personal files, but there is more to this. Security ensures that there is no damage to your data and that only authorized users can read your files. This work focuses on the dimension of remote controlled alert system for automobile access using password credential. This remote control alert was done by research on how remote controlled systems and automatic gates work and the transfer of frequency signals through transmitters to receiver module to be used at homes, schools, industries and companies. The remote alert system was developed by combining major systems: the power generation system or power source, transmission system, reception system (the alert system, the actuation system and the display system. Results showed that at smaller distance of $15 \mathrm{~cm}$, the response time was $0.12 \mathrm{~s}$ while at a longer distance of $100 \mathrm{~cm}$; the response time was $15.91 \mathrm{~s}$. A valid signal was received on the receiver output display for automobile access into a premise; therefore TLP 315MHz RF transmitter and the RLP $315 \mathrm{MHz}$ RF receiver can be used for a shorter distance.
\end{abstract}

Keywords: Automobile, Microcontroller, Receiver, Sensor, Transmitter

\section{Introduction}

An alert system is a mechanical, electromechanical or piezoelectric system that gives an audible, visual or other form of alarm signal about a problem or condition. These alarm signals are often incited by electronic signals which are generated by electronic relay, photoelectric sensors, or any power source. They are often outfitted with a siren. This system deals with access control as regard to the entry of an individual or vehicle into given vicinity. They are developed to avoid theft (Fan and Zhang, 2009), to enhance access control to car parking (Hsieh et al., 2008; Sheelaet al., 2014). It allows users to locate vehicle and perform other actions like opening of doors without key or starting engine remotely (Sanchez et al., 2013; Levelna, 2012; Tracer, 2012). An individual possessing the password that is programmed to the device is able to have access only into the area or premises thereby prohibiting entry of another individual without due authorization(Calson, 2006). This device can be used in a number of places such as hotels, banks, organizations, homes, and schools.

A remote controlled alert system is basically a system, in which the audio or visual signal given by the alert system influences the operation of a remote or push button to actuate or incite an action such as to push or pull, to on or off a switch, to open or close a gate or any action of the user's choice. A remote controlled alert system for vehicle access into a premise is an alert system which is triggered on receiving an electric signal from a transmitter (remote) on vehicle proximity, and consequently influences the actuation of an open or close of a gate or access barrier, by a push button or remote control. Remote control vehicles have various scientific uses including hazardous environments, working in the Deep Ocean, and exploration. RCV is used by police to detect bombs, or some chemicals. It detects chemicals its high-power sensors. The majority of the probes to the other planets in our solar system have been remote control vehicles, although some of the more recent ones were partially autonomous. The sophistication of these devices fueled greater debate on the need for manned spaceflight and 
exploration. The Voyager I spacecraft is the first craft of any kind to leave the solar system. According to Finn et al. (2010), the Martian explorers Spirit and Opportunity have provided continuous data about the surface of Mars since January 3,2004. The proposed infrared remote controlled door lock circuit can be used for locking your main door, gate, garage door, shop or any entrance which may need a foolproof internal locking through a remote control system (Mujumdar, 2015). Iceman worked on the development of the working push-pull amplifier, none of the circuits was able to drive the car both in the forward and reverse directions. One of the circuits can be pulse width modulated between fast and very fast, but could not be turned off. The lesson learned is that the trick to working with BJTs is having a very tight control over the base currents. Again, power MOSFETs would have made this significantly easier (Iceman, 1999). This work is done to demonstrate the principle and operation of a remote controlled alert system for vehicle access into a premise. The equipment used in ultimately realizing the finished product was in line with Smart Card Handbook (Rankl and Effing, 1997). The aim of this work is to design a remote controlled alert system for vehicle access into premise, fabricate the transmitting, alarming, sliding gate control system and evaluate the fabricate the remote controlled alert system for vehicle access.

\section{Methodology}

The block diagram in Figure 1 shows various components used in the development of the remote controlled system:

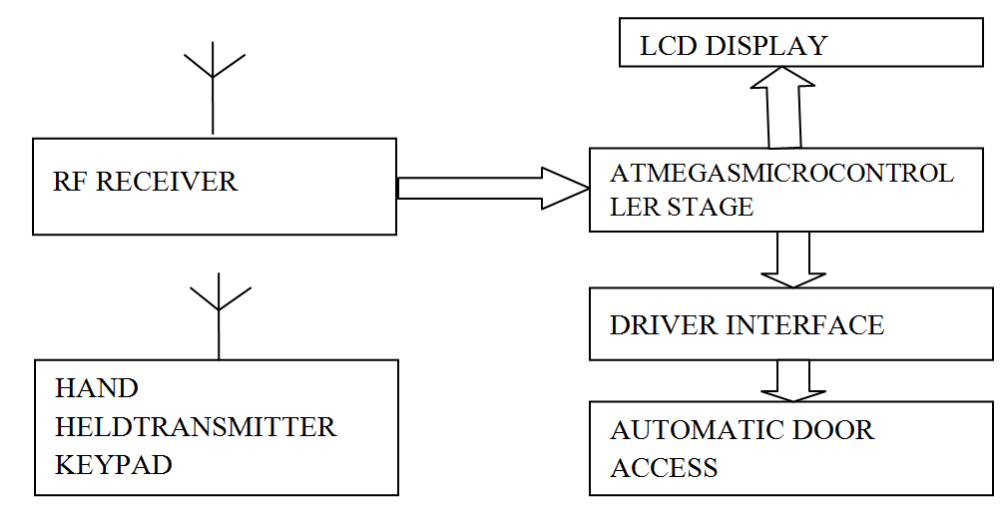

Figure 1. Generalized block diagram for remote controlled alert system for automobile access.

The remote alert system was made by combining major systems: The power generation system or power source, transmission system, reception system (the alert system, the actuation system and the display system.

The power source or power generation system generates power for the transmission and reception system. This power source is different for the three systems: an a.c (alternating current) source and a dc source. For the purpose of achieving the desired result for this project, a DC (Direct Current) power source for the transmission system and an a.c source for the reception, alert and actuation system was used. The power supply stage provides the appropriate DC voltage requirements to ensure the circuit components are powered properly. The ATMEGA8 requires a maximum voltage of $+5.5 \mathrm{~V}$, which makes $5 \mathrm{~V}$ power supply okay for the circuit. The power supply stage is a linear power supply type and involves in step down transformer, rectifier, filter capacitor and voltage regulators, to give the various voltage levels. The transmission system comprises the electrical components that influence the remote control to transmit signals through radio frequency signals to the radio frequency receiver. The components of the transmission system are simply the components of the remote control. These white box components are: $9 \mathrm{~V}$ dc source, $470 \mu \mathrm{F}$ capacitor, ATMEGA8 microcontroller, Push button (12), HT12E RF encoder, RF transmitter, 10k Resistor, Antenna, Electronic chip board, Transparent plastic 8 by $4 \mathrm{ft}$ for housing, LCD LED (2)

The remote controlled alert system was put into realization to produce its desired output result. The use of the cost effective components like the TLP RF transmitter, RLP RF receiver of $315 \mathrm{MHz}$ each, the ATMEGA8 microcontroller, the HT12E encoder, HT12D decoder, a simple buzzer, the resistors, capacitors, LEDs and LCDs in place of more advanced but less cost effective (which would still yield the same results for the prototype project) like Arduino UNO or NANO, PIC18F452 microcontroller, 433MHz RF receivers and transmitters were all connected together.

\subsection{Design and Fabrication the Remote Control}

The design and construction of the remote control is done by careful and scrupulous measures, working according to design and calculation in order to avoid errors, accidents and system failures. It is first designed in Autodesk inventor, the physical or white box model and then the circuit is designed in Proteus 8.1 ISIS library. All work is done according to developed designs in both fields. The remote control transmitter module operating at $315 \mathrm{MHz}$ frequency using complex transmission for purpose of sending the code to the receiver while the receiver listens for the code. The ATMEGA8 microcontroller is the main processing unit for this project. Its job is to perform timing and control functions accurately, as well as encode and decode the signal to be sent and received from the transmitter to the receiver in the reception system, to also control the rotation of the servomotor for door opening and closing in the actuation 
subsystem. The ATMEGA 8 has $1 \mathrm{~K}$ of program memory and 23 programmable I/O lines (Calson, 2006). The chip board is cut into desired size: a length of $8 \mathrm{~cm}$, and a width of $3.5 \mathrm{~cm}$. The chip board is cut with a metallic cutter, after points are outlined on the chip board and then cut. A metre rule is placed beside the metallic cutter to guide it while the cutting is done. The transparent plastic is cut to size: a length of $110 \mathrm{~mm}$, breadth of $40.5 \mathrm{~mm}$ and a thickness of $2 \mathrm{~mm}$. this is also done with the metallic cutter for easy and sharp cuts, guided by a metre rule. The circuit diagram of the remote control is shown in Figure 2.

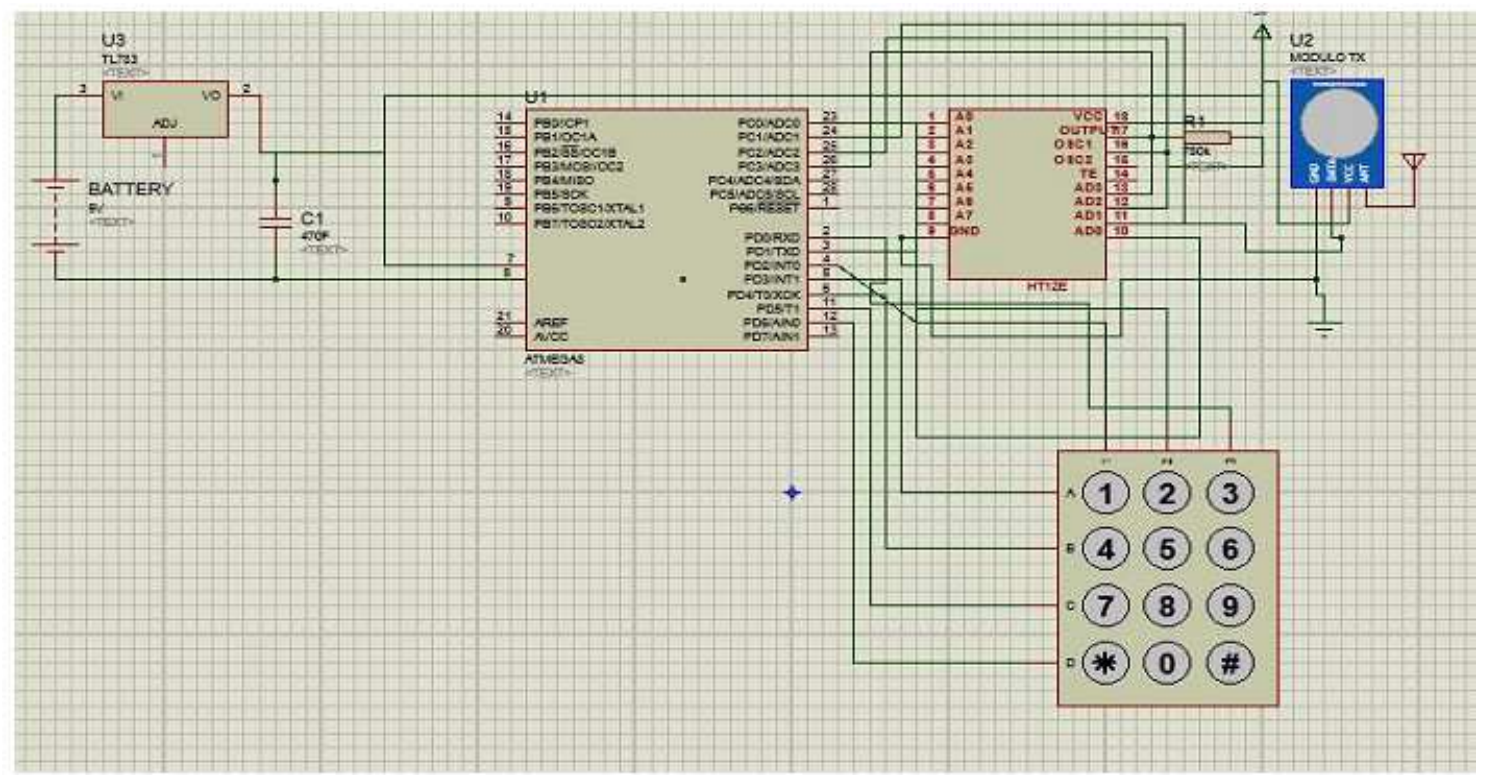

Figure 2. Circuit diagram of remote control using Proteus 8.1.

\subsection{Reception System}

This system is the other major system in this project as it comprises all the electrical and mechanical components necessary for converting the transmitted signals into an audio signal and kinetic energy (actuation). The system is fabricated according to the laid out engineering design, under careful and scrupulous actions. The components of the reception system: $220 \mathrm{~V}$ AC, transformer, rectifier, $7805 \mathrm{~V}$ voltage regulator, $470 \mu \mathrm{F}$ capacitor (smoothener), $220 \mu \mathrm{F}$ capacitor (filter), $100 \Omega$ resistor, $1 \mathrm{~K}$ resistor, ATMEGA8 microcontroller, HT12D RF decoder, RLP 315 RF receiver, Antenna, LCD, Buzzer. As stated earlier, the power supply stage is a linear power supply type and involves in step down transformer, rectifier, filter capacitor and voltage regulators, to give the various voltage levels. The circuit diagram of the receiver power supply stage is shown in Figure 3.

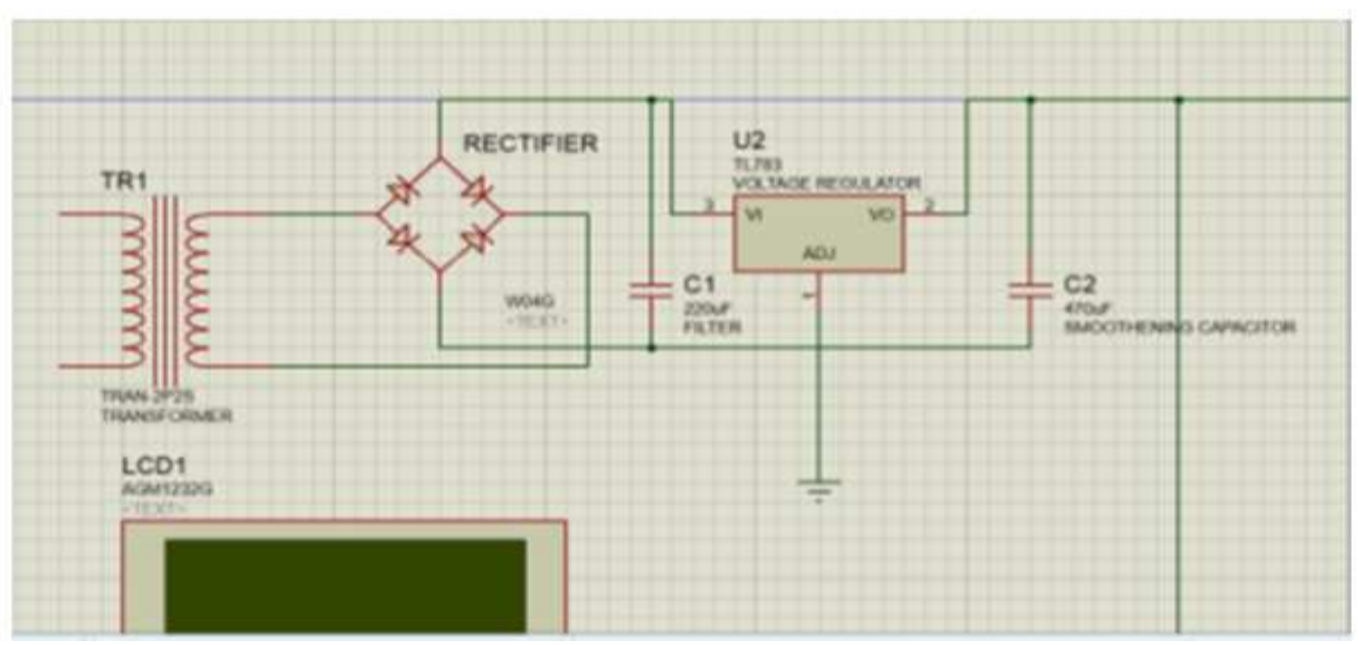

Figure 3. Circuit diagram of the receiver power supply stage.

The voltage regulator is used to stabilize the voltage after it has been filtered. In this case, the output voltage of $14 \mathrm{~V}$ filtered by the capacitor is then stabilized to a usable working voltage of $5 \mathrm{~V}$ which is the required voltage utilized by the
ATMEGA8 microcontroller. The system, since it gives two major forms of output signals (audio signal and actuation), we can say it is subdivided into two systems:

1. The alert system 
2. The actuation system

Specifications of the design are drawn out and clearly stated in detail in the diagrams below:

Model: rectangular box; material: plastic; thickness: $2 \mathrm{~mm}$; side size: $50.5 \mathrm{~mm} \times 70.5 \mathrm{~mm}$; top size: $100 \mathrm{~mm} \times 70.5 \mathrm{~mm}$.

The circuit diagram of the reception system is shown in Figure 4.

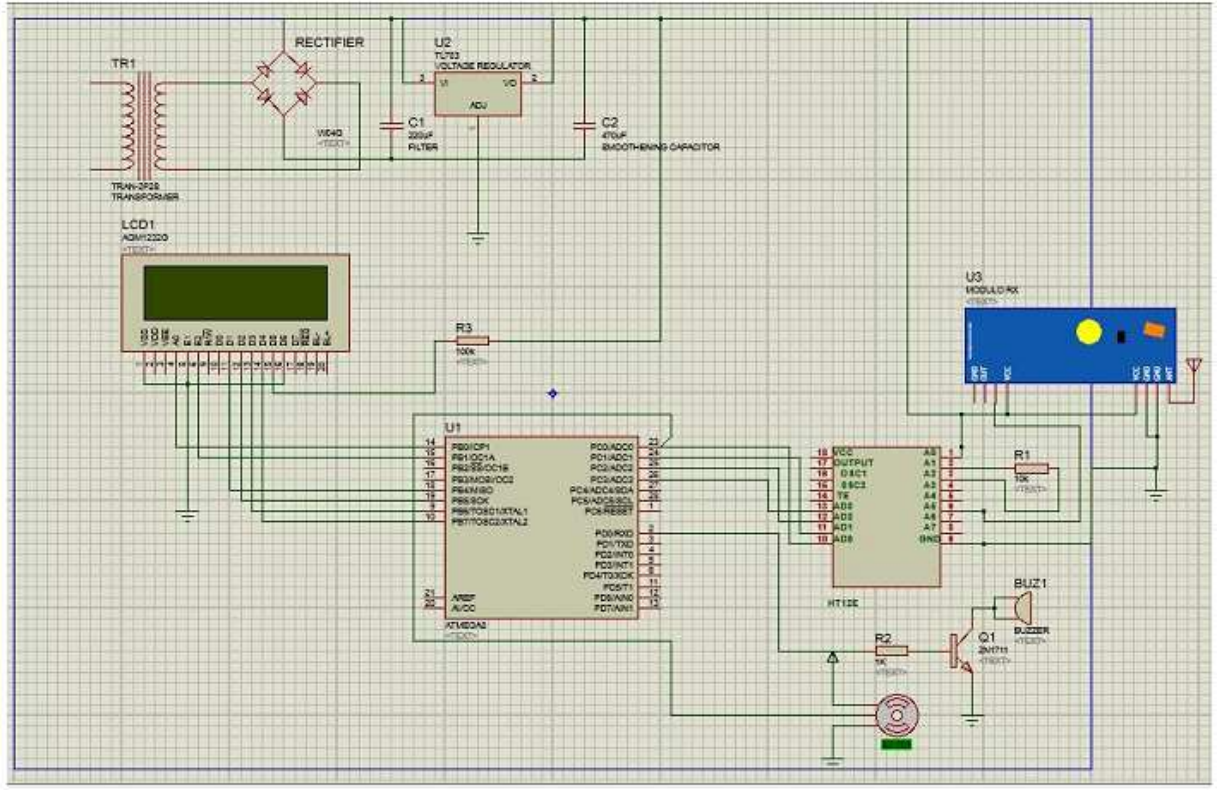

Figure 4. The circuit diagram of the reception system.

\subsection{The Alert System}

The alert system is responsible for converting the output from the decoder to an audio signal by the control function of the ATMEGA8 microcontroller to the transistor to which the audio device is connected. The device used for giving out the audio signal in this project is a simple buzzer. Transistor is a bipolar transistor, which acts as a switch for the buzzer. The transistor is connected to the PORT D (PD0/RXD) which generates commands in programmed pulses through a $1 \mathrm{~K}$ resistor, the timed pulses are converted to timed audio signals.

\subsection{The Actuation System}

This system basically converts the source from PORT D of the ATMEGA8 microcontroller into a sliding motion of the prototype gate. The conversion of the electronic signal or code into physical action is aided by the tower pro MG996R metal gear digital torque servo and the sliding door mechanism. The servo motor is powered by the low current source of the PORT D from the ATMEGA8 microcontroller which causes its rotor to rotate at $0.04 \mathrm{~A}$, angle of $180^{\circ}$, dead zone of 2 microseconds and a torque of $9.4 \mathrm{~kg} / \mathrm{cm}$ (Jerry, 2001). This motion is converted into linear motion by the sliding door mechanism which causes the door to slide $180^{\circ}$ forward, as an "open" action or $180^{\circ}$ backwards, as a "close" action for vehicles access into the premises. The sliding door mechanism consists of two rectangular cuts of wood of length $300 \mathrm{~mm}$, groove depth of $4 \mathrm{~mm}$, groove width of $2 \mathrm{~mm}$ and $8 \mathrm{~mm}$ thickness; a model gate of length of $200 \mathrm{~mm}$ and width of $400 \mathrm{~mm}$, with a rectangular slut of $180 \mathrm{~mm}$ and width of $10.5 \mathrm{~mm}$, to which a slut pin of length $6 \mathrm{~mm}$, diameter of $10 \mathrm{~mm}$, pin cap of thickness $10 \mathrm{~mm}$ and diameter $15 \mathrm{~mm}$, which is also connected to the circumference of a circular plate of diameter $100 \mathrm{~mm}$, thickness of $10 \mathrm{~mm}$, having a through hole at its centre of $2 \mathrm{~mm}$ diameter, a groove of $4 \mathrm{~mm}$ diameter and depth of $6 \mathrm{~mm}$ also at its centre.

The tower pro MG996R metal gear digital torque servo rotates $180^{\circ}$ according to the ATMEGA8 program after the security pin is typed on the remote control, causing the circular plate to rotate the slut pin; the pin consequently causes a "push" motion to the sliding door to which it is connected (circular to linear motion conversion). Later on, the tower pro MG996R metal gear digital torque servo rotates $180^{\circ}$ in reverse motion according to the ATMEGA8 program, causing a "pull" action which closes the model gate.

\section{Results}

The result obtained from testing the remote controlled alert system for vehicles access to judge its response time at different distances compared to its expected distance range is presented in Table 1.

Table 1. Relationship between distance and response time.

\begin{tabular}{lll}
\hline $\mathbf{S} / \mathbf{N}$ & Distance $(\mathbf{c m})$ & Response time (s) \\
\hline 1 & 15 & 0.12 \\
2 & 30 & 0.41 \\
3 & 50 & 0.43 \\
4 & 100 & 15.91 \\
\hline
\end{tabular}

From Figure 2, the relationship between the distance value 
of the two major systems, transmitter and the receiver and the response time value at different distances as tested can be seen. From the results obtained, we can infer that the TLP $315 \mathrm{MHz}$ RF transmitter and the RLP $315 \mathrm{MHz}$ RF receiver are not reliable for long distant communications. However, for shorter distances as shown in Table 1, the TLP $315 \mathrm{MHz}$ RF transmitter and the RLP $315 \mathrm{MHz}$ RF receiver can be used.

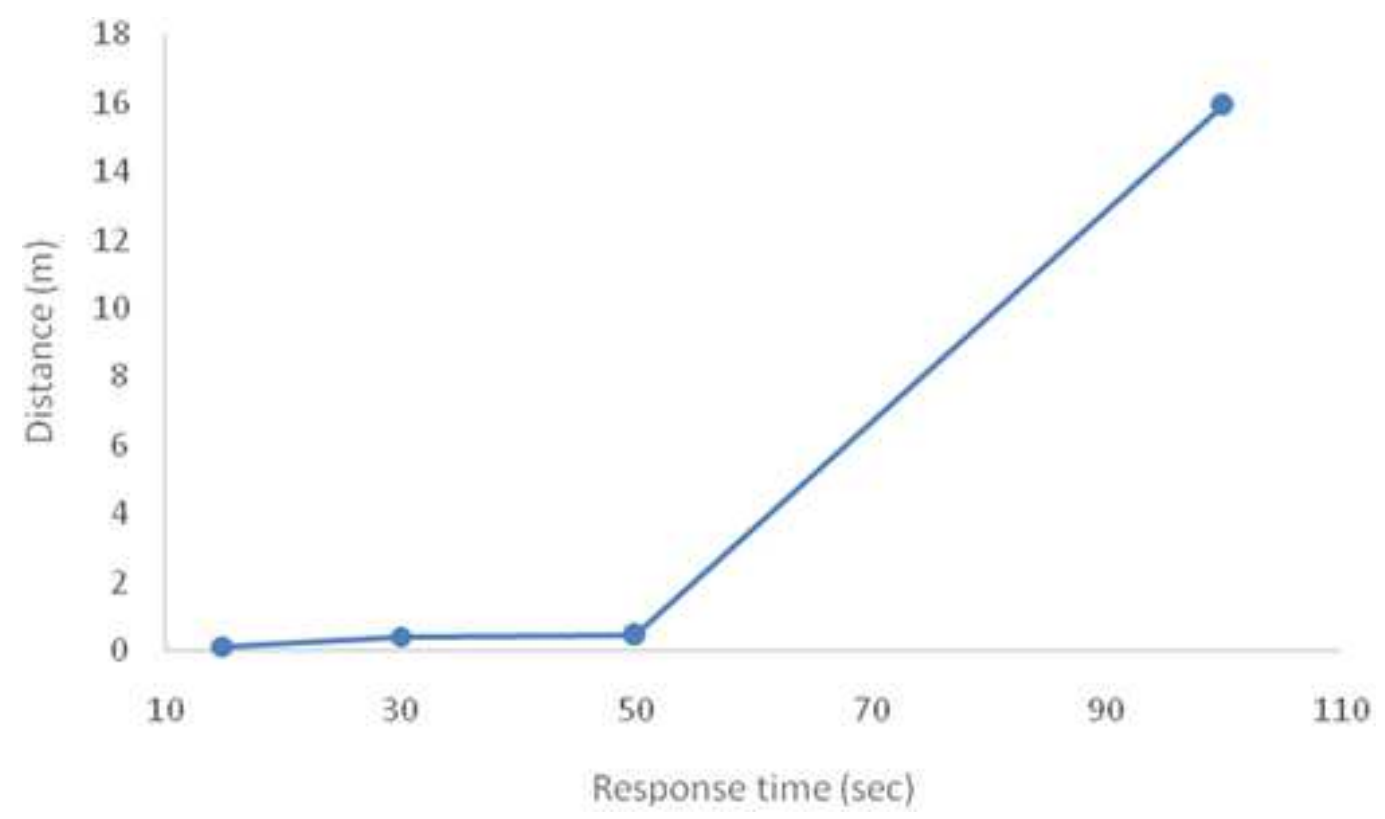

Figure 5. Relationship between distance and response time.

\section{Discussion}

Figures 6 and 7 show the different display outputs on the receiver for different input signals. The figure 6 displays the receiver output when it is connected to a power source and figure 7 shows the receiver display output when the input four-digit security code is typed on the remote control by the user to actuate the sliding door mechanism.

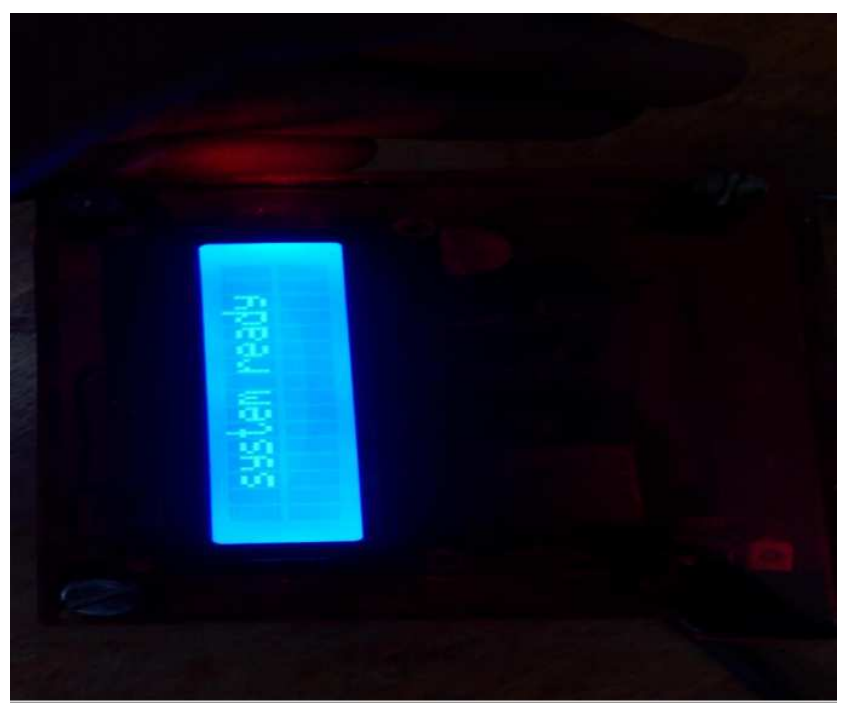

Figure 6. Receiver output display.

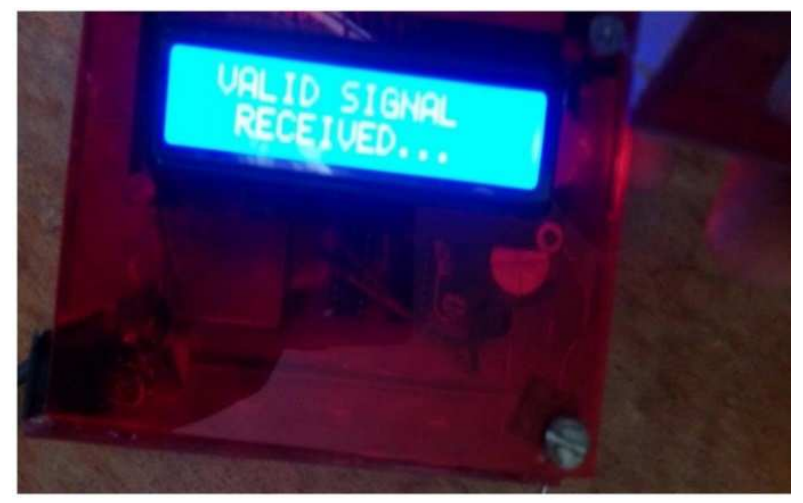

Figure 7. Receiver output display.

\section{Conclusion}

This work is an answer to the demand for more effective security by individuals, companies, schools and the society taken as a whole and has evolved the norm of having a key or and RFID (Radio Frequency Identification) tag to the use of pass code as its unique credential which is peculiar to the individual. We have been able to achieve a complete working system made of two major units: the transmitter and receiver unit also having a sliding door mechanism, operated by a code, without which there is no entry. The remote controlled alert system for automobile access into a premise is fabricated: its transmitting, alarming and sliding gate control system is fabricated and tested to be working.

The work study done on the remote controlled alert system for automobile access into premise shows that it is a workable system and cost effective, hence the detailed 
components and the working principle as well as mechanisms used for this project are recommended for system involving access security or access control.

\section{Recommendations}

However, developments can be made to required components of the remote controlled alert system for automobile access into a premise, depending on the required capacity of the system. Parameters can be altered but not necessarily changed for this to work in either small or largescale system of this kind.

\section{References}

[1] Calson, (2006). "Surveillance and Identification: Identity", Calson Analytics research, analysis and strategies consultancy.

[2] Fan, X. H. and Zhang, Y. L. A. (2009). Design of Biverification Vehicle Access Intelligent Control System based on RFID. Proceeding, $9^{\text {th }}$ Int'l. Conference on Electronic Measurement and Instruments (ICEMI 2009) Beijing China, Aug. 16-19 2009.

[3] Finn, Scheding, A. and Steve (2010). Developments and Challenges for Autonomous Unmanned Vehicles: A Compendium. Volume 3: Intelligent Systems Reference Library. Springer Science \& Business Media. pp. 7. ISBN 36421027044.

[4] Hsieh, W. H., Ho, C. J. and Jong, G. J. Vehicle Information Communication Safety Combined with Mobile RFID. Proceeding, Int'l Conference on Intelligent Information
Hiding and Multi-media Signal Processing, Harbin China, Aug. 15-17, 2008.

[5] Iceman, D. (1999). EE 476 Final Project. Retrieved June 21st, 2016 ,

frompeople.ece.cornell.edul://people.ece.cornell.edu/land/cour ses/ece4760/FinalProjects/s1999/blair/RCar.html.

[6] Landt, Jerry (2001). "Shrouds of Time: The history of RFID", 2001.

[7] Levelna (2012). Car Alarm, GSM, GPRS. User Guide to Installation and Operation Level System. Available at $\mathrm{http}: / / w w w . l e v e l n a . c o m p o s i t r e x$. Retrieved on 22-01-2016.

[8] Majumdar, S. (2015). Infrared Remote Controlled Door Lock Circuit. Retrieved June 21st, 2016, from home madecircuits.com: http//: home made circuits and schematics. Available at blogspot.in/2015/02/infrared-remote-controlsecurity-lock.html. Retrieved on 2-3-2016.

[9] Rankl, W. and Effing, W. (1997). Smart Card Handbook. John Wiley and Sons.

[10] Sanchez, J. M., Heredia, O. S. and Lopez, E. V. (2013). Development of a Vehicle Security System with Local and Remote Control and Event Notification. Redes de Ingenieria 4(2) 1-7.

[11] Sheela, J., Akil, S., Akil, K., Narayanankutty, Y., Amalraj, P. M. and Aleena, P. K. (2014). RT Transceiver Based Traffic Alert System for Automobile. Int'l Journal of Advanced Research in Electrical Electronics and Instrumentation Engineering. 3(2)7623-7627.

[12] Tracer (2012). Trace Product Overview. available at hhttp://wwwewcop.com. Retrieved on 22-01-2016. 